\title{
Is there an excess risk for colorectal cancer in patients with ulcerative colitis and concomitant primary sclerosing cholangitis? A population based study
}

D Kornfeld, A Ekbom, T Ihre

\begin{abstract}
Background-Patients with ulcerative colitis have an increased risk of colorectal cancer. Duration, age, and extent of the disease at diagnosis are the only established risk factors. Patients with ulcerative colitis and concomitant primary sclerosing cholangitis (PSC) have been reported to have a higher frequency of colonic DNA aneuploidy and/or dysplasia than expected, findings indicating an increased risk of colorectal cancer compared with other patients with ulcerative colitis.

Methods-A population based cohort consisting of 125 patients with a verified diagnosis of PSC was followed up by linkage to the Swedish Cancer Registry for the occurrence of colorectal cancer.

Results-There were 12 colorectal cancers. Six cancers were diagnosed prior to the diagnosis of PSC. Among the 104 patients with an intact colon at the time of the diagnosis of PSC there was a cumulative risk for colorectal cancer of $16 \%$ after 10 years. Among the 58 patients with a diagnosis of ulcerative colitis and colorectal cancer prior to the diagnosis of PSC, there were five colorectal cancers corresponding to a cumulative risk of $25 \%$ after 10 years.

Conclusions-Patients with ulcerative colitis and concomitant PSC seem to constitute a subgroup with a high risk for colorectal cancer.

(Gut 1997; 41: 522-525)
\end{abstract}

Keywords: ulcerative colitis; primary sclerosing cholangitis; colorectal cancer

Department of Cancer

Epidemiology,

University Hospital,

S-751 85 Uppsala,

Sweden

D Kornfeld

A Ekbom

Department of

Surgery,

Södersjukhuset,

Stockholm, Sweden

T Ihre

Correspondence to:

Dr D Kornfeld.

Accepted for publication 25 March 1997

Patients with ulcerative colitis have an increased risk of colorectal cancer. The magnitude of this risk varies in different studies with a range in cumulative risk of between $1 \%$ and $3 \% 10$ years after diagnosis in patients with pancolitis and around $10 \%$ after 20 years. ${ }^{1-6}$ Duration, age, and extent of the disease at onset are the only established risk factors for malignant transformation. ${ }^{1}$ Recently published studies also indicate that patients with ulcerative colitis who have had no pharmacotherapy, have an increased risk for colorectal cancer compared with patients treated with sulphasalazine..$^{7-9}$ This could indicate that an inflammatory component is of importance for malignant transformation. Patients with ulcerative colitis with a diagnosis of primary sclerosing cholangitis (PSC) constitute another subgroup which also has been implicated to be at especially high risk for colorectal cancer, DNA aneuploidy, and/or colonic dysplasia, ${ }^{10-14}$ although such an association has recently been challenged. ${ }^{15}$

The majority of patients with PSC also have an associated inflammatory bowel disease, especially ulcerative colitis. ${ }^{16}$ The occurrence of PSC in patients with ulcerative colitis is dependent on the extent of the disease..$^{15} \mathrm{~A}$ population based Swedish study found a prevalence of $5.5 \%$ in patients with substantial colitis and $0.5 \%$ in patients with distal colitis. ${ }^{17}$ These estimates have been confirmed by other studies from referral centres where a similar prevalence of about $5 \%$ has been found. ${ }^{18} 19$

All reports indicating that patients with ulcerative colitis and concomitant PSC are at especially high risk for colorectal cancer emanate from referral centres and valid comparisons with other patients with ulcerative colitis are lacking. Thus, in order to provide reliable risk estimates we conducted a population based cohort study of patients with PSC followed up for the occurrence of colorectal cancer.

\section{Methods}

The Swedish Health Care system is based on 26 counties. Health care is largely financed by taxes imposed by the county and charges for medical services are kept low. Because the private hospital sector is very small in Sweden, hospital provided medical services are population based and referable to a county.

Endoscopic retrograde cholangiopancrea- tography (ERCP) was gradually introduced in Sweden in the early 1970 s. We identified seven hospitals with a background population of 990000 people, where the method was introduced at an early stage. In one of those hospitals, South Hospital in Stockholm, PTC (percutaneous transhepatic cholangiography) had been used routinely as a diagnostic tool since 1964. Through an extensive search in the archives in these seven different hospitals between the years 1964-91 we identified all patients which had been subjected to an ERCP or PTC within each catchment area (approximately 5000), thus creating a population based 
TABLE 1 Age at diagnosis of PSC in 125 patients and duration between the diagnoses of ulcerative colitis and PSC in 79 patients with a prior diagnosis of ulcerative colitis

\begin{tabular}{llll}
\hline & Men & Women & All \\
\hline Age (y) at PSC diagnosis & $41.5(16-76)$ & $48(14-79)$ & $43.5(14-79)$ \\
Duration (y) between diagnoses & $15.3(1-48)$ & $19.5(1-43)$ & $16.5(1-48)$ \\
\hline
\end{tabular}

cohort of all incident and prevalent PSC cases within a defined population.

All Swedish residents are exclusively identified by a 10 digit national registration number. Through this identifier we retrieved all patient charts of those with a suspected diagnosis of PSC. From the charts we retrieved information on medical history including a prior or later diagnosis of inflammatory bowel disease and if and when a patient had had a colectomy.

The Swedish National Cancer Registry has been in operation since 1958. Physicians and pathologists or cytologists are obliged to report all newly diagnosed malignant tumours, making the Registry almost complete. ${ }^{20}$ At the time of this study the Registry was complete as of 31 December 1991, which was the closing date of follow up. The Registry of Causes of Death includes the date of death for all deceased persons as well as the underlying cause of death. It contains information on individual deaths in Sweden from 1952. The deceased has to be a Swedish resident and all deaths are registered irrespective of whether or not the death occurs in Sweden. Through linkage with the Swedish Cancer Registry and the Death Registry all cases of colorectal cancer were identified as well as the date of the diagnosis of cancer and date of death.

In order to calculate the cumulative risk of colorectal cancer starting at time of the diagnosis of PSC, we used the actuarial method (Kaplan-Meier) censoring for death and colectomy. ${ }^{21}$ In a further analytical approach, the duration of ulcerative colitis before the diagnosis of colorectal cancer was analysed and patients were considered to be at risk for colorectal cancer only after the diagnosis of PSC.

\section{Results}

We identified 125 patients who fulfilled the criteria of PSC verified by ERCP or PTC between the years 1964-91. There were 82 men and 43

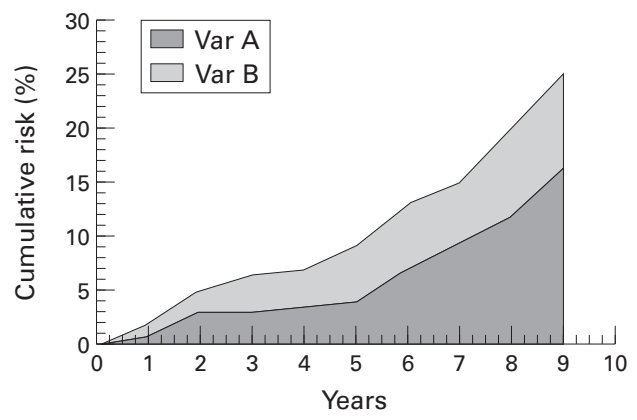

Figure 1: Var A: cumulative risk for colorectal cancer in 104 patients with PSC and intact colon. Six colorectal cancers were available for analysis. Var B: cumulative risk for colorectal cancer in 58 patients with ulcerative colitis prior to PSC diagnosis and intact colon. Five colorectal were cancers available for analysis.

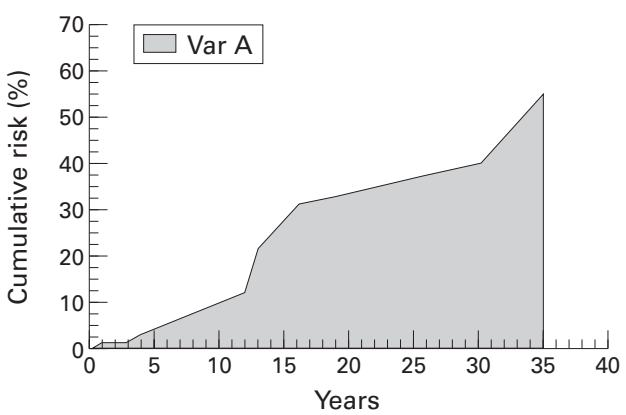

Figure 2: Cumulative risk for colorectal cancer in patients with ulcerative colitis and PSC by duration since the diagnosis of ulcerative colitis.

women. Table 1 presents age at PSC diagnosis in all patients and duration between ulcerative colitis and PSC diagnosis in 79 patients with a prior diagnosis of ulcerative colitis.

In 79 patients, inflammatory bowel disease was diagnosed prior to PSC. In 75, the inflammatory bowel disease diagnosis was stated as ulcerative colitis; in four the diagnosis was either categorised as indeterminate colitis or suspected Crohn's disease. All 79 patients will, however, be analysed as one entity. The mean age at diagnosis of PSC for ulcerative colitis patients was 39.2 years. This group was younger than those with PSC without ulcerative colitis (52.2 years). In addition six patients were diagnosed as having ulcerative colitis subsequent to the PSC diagnosis. Twenty one patients had had a colectomy before the diagnosis of PSC. Through linkage with the Swedish Cancer Registry 12 colorectal cancers were identified. Six of those cancers were diagnosed prior to diagnosis of PSC which in all instances had lead to a proctocolectomy. Among the 104 patients with PSC and intact colon available for follow up there was a cumulative risk of $16 \%$ for colorectal cancer 10 years after the diagnosis of PSC (fig 1, var A). Confining the analysis to those 58 patients with a prior diagnosis of ulcerative colitis and with an intact colon, there were five colorectal cancers corresponding to a cumulative risk close to $25 \% 10$ years after diagnosis of PSC (fig 1, var B).

In a second analytical approach, the cumulative risk of colorectal cancer was assessed by duration since date of diagnosis of ulcerative colitis. In this analysis the patients were regarded as being at risk only after the date of diagnosis of PSC. The cumulative risk of colorectal cancer was $10 \%, 33 \%$, and $40 \%$, respectively, 10, 20, and 30 years after the diagnosis of ulcerative colitis (fig 2).

\section{Discussion}

This study is the first attempt to assess the magnitude of the risk of colorectal cancer in patients with ulcerative colitis and PSC in a population based setting. Our results indicate that this patient group constitutes a subgroup of patients with ulcerative colitis with an especially high cumulative risk for colorectal cancer. These results are in accordance with other studies where a slightly different outcome, such as DNA aneuploidy and/or 
dysplasia, has been analysed. ${ }^{11}$ It is also obvious from our results that the increased risk for colorectal cancer in patients with PSC is confined to those with a diagnosis of inflammatory bowel disease.

Our statement that patients with ulcerative colitis and PSC are at especially high risk for colorectal cancer might be questioned due to the lack of a well defined control group of patients with ulcerative colitis. For comparison purposes our study therefore has to rely on other studies from different populations or time periods for the assessment of the risk of colorectal cancer. ${ }^{1}$ The cumulative risk of colorectal cancer in patients with ulcerative colitis 10 years after diagnosis has been estimated at most to increase $10-15 \%$ every additional decade following diagnosis. ${ }^{122} 23$ Thus, our finding of a cumulative risk of $25 \%$ during the first 10 years after the diagnosis of PSC (fig 1, var B) is unprecedented in previous studies. However, when the duration of ulcerative colitis is analysed as the relevant exposure in the present study (fig 2) the cumulative risk of $33 \%$ after 20 years is high but not unprecedented compared with some other studies. $^{24}$ The recent study from the Mayo Clinic did not find an increased risk for colorectal cancer among patients with ulcerative colitis and PSC compared with other patients with ulcerative colitis. In addition to the low statistical power, the fact that the study emanated from a single referral centre could at least partially explain the differences in the results. Patients from a single referral centre might be subjected to an intensified colonoscopic surveillance, and thus a higher rate of colectomy ( $37 \%$ versus $17 \%$ in our study). ${ }^{24}$

The main weakness of this study is the small number of patients enrolled; the calculation of the cumulative risk of colorectal cancer in patients with ulcerative colitis emanates from only five cases of cancer. On the other hand, the strength of this study is the fact that it is population based and the methodology used by creating a cohort from ERCP and PTC registries minimises the risk of selection bias. Moreover, the follow up through the Swedish Cancer Registry is non-differential and there is no reason to believe that surveillance bias with regard to occurrence of colorectal cancer should be a concern compared with other patients with ulcerative colitis.

Another interesting finding in our study was the presence of six colorectal cancers diagnosed prior to the diagnosis of PSC in the group of patients with ulcerative colitis. Selection bias could be a concern in this group as the follow up of patients who received surgery for colorectal cancer often includes repeated tests of liver function and thus perhaps an earlier diagnosis of PSC. On the other hand, mortality from colorectal cancer in patients with ulcerative colitis does not differ from that of other patients with colorectal cancer. Around $50 \%$ of patients diagnosed with colorectal cancer will succumb to the disease within five years. ${ }^{25}$ This excess mortality could lead to an underestimation of PSC in patients with ulcerative colitis and colorectal cancer as they will not survive until a potential diagnosis of PSC. The excess number of colorectal cancers diagnosed before the diagnosis of PSC diagnosis indicates that it is not the PSC diagnosis as such which is associated with an increased risk of colorectal cancer in this patient group. A more feasible explanation is that this subgroup of patients has a common feature resulting in an increased risk of both PSC and colorectal cancer.

The underlying biological mechanism for the association of PSC and colorectal cancer is still unknown. However, proctocolectomy in patients with ulcerative colitis does not alter the course of PSC $^{26}$ and the risk of colorectal dysplasia/cancer does not seem to be influenced by liver transplantation in patients with PSC and ulcerative colitis. ${ }^{27}$ Patients with ulcerative colitis and PSC are also often characterised by a disease with low activity or even a "silent" disease. ${ }^{13}{ }^{16}$ It is not uncommon that the diagnosis of ulcerative colitis is made simultaneously or even after a diagnosis of PSC, as in the present study. This patient group will therefore be subjected to pharmacological therapy less frequently than other patients with ulcerative colitis. Pharmacological therapy, especially sulphasalazine, has been shown to be associated with a decreased risk of colorectal cancer both indirectly ${ }^{8}$ and directly, ${ }^{7}$ and could at least partly explain the high risk of colorectal cancer in this patient group.

Finally, it is not inconceivable that a cohort of patients with ulcerative colitis does not constitute one disease entity. However, there is a strong association between ulcerative colitis and PSC and a group of patients with both diseases will therefore constitute a "pure" group of patients with ulcerative colitis. If the risk estimates for colorectal cancer in patients with ulcerative colitis have emanated from patient groups diluted by other diseases mimicking ulcerative colitis, or if ulcerative colitis is a mixture of different disease entities in which only some are associated with an increased risk for colorectal cancer, this could also, at least partly, explain our findings of an excess morbidity in colorectal cancer in patients with ulcerative colitis and concomitant PSC.

In conclusion, patients with ulcerative colitis and concomitant PSC seem to constitute a subgroup of patients with ulcerative colitis with an especially high risk for colorectal cancer. This patient group should therefore be informed and targeted for surveillance, thus minimising the risk for early death from this malignancy.

\footnotetext{
1 Ekbom A, Helmick C, Zack M, Adami H-O. Ulcerative colitis and colorectal cancer; a population-based study. $N$ Engl f Med 1990; 323: 1228-33.

2 Kewenter J, Ahlman H, Hulten L. Cancer risk in extensive ulcerative colitis Ann Surg 1978; 188: 824-8.

3 Maratzka Z, Nebal J, Kocianova J, Halevka J, Kurdman J, Hendl J. Incidence of colorectal cancer in proctocolitis: a retrospective study of 959 cases over 40 years. Gut 1985; 26: $43-9$.

4 Katzka I, Brody R, Morris E, Katz S. Assessment of colorectal cancer risk in patients with ulcerative colitis: experience from a private practice. Gastroenterology 1983; 85: 22-9.

5 Gilat T, Fireman Z, Grossman A, Hacohen D, Kadish E, Ron E, et al. Colorectal cancer in patients with ulcerative colitis. Gastroenterology 1988; 94: 870-7.

6 Whelan G. Ulcerative colitis-what is the risk of developing colorectal cancer? Aust NZ Med F 1991; 21: 71-7.
} 
7 Pinczowski D, Ekbom A, Baron J, Yuen J, Adami H-O. Risk factors for colorectal cancer in patients with ulcerative colitis: a case-control study. Gastroenterology 1994; 107: $117-20$.

8 Langholz E, Munkholm P, Davidsen M, Binder V. Colorectal cancer risk and mortality in patients with ulcerative colitis. Gastroenterology 1992; 103: 1444-51.

9 Lynch DAF, Lobo AJ, Sobola GM, Dixon MF, Axon ATR. Failure of colonoscopic surveillance in ulcerative colitis Gut 1993; 34: 1075-80.

10 D'Haens GR, Lashner BA, Hanauer SB. Pericholangitis and sclerosing cholangitis are risk factors for dysplasia and cancer in ulcerative colitis. Am $\mathcal{F}$ Gastroenterol 1993; 88: 1174-8.

11 Broomé U, Lindberg G, Löfberg R. Primary sclerosing cholangitis in ulcerative colitis - a risk factor for the development of dysplasia and DNA aneuploidy? Gastroenterology 1992; 102: 1877-80.

12 Hanauer S. Primary sclerosing cholangitis and ulcerative colitis: potential cofactors in the dysplasia sequence. colitis: potential cofactors in the

13 Gurbuz KB, Giardiello FM, Bayless TM. Colorectal neoplasia in patients with ulcerative colitis and primary

14 Brentall TA, Haggitt RC, Rabinovitch PS, Kimmey MB Bronner MP, Levine DS, et al. Risk and natural history of colonic neoplasia in patients with primary sclerosing cholangitis and ulcerative colitis. Gastroenterology 1996; 110: $331-8$

15 Loftus EV, Sandborn WJ, Tremaine WJ, Mohoney DW, Zinsmeister AR, Offord KP, Melton LJ. Risk for colorectal cancer in patients with primary sclerosing cholangitis. Gastroenterology 1996; 110: 432-40.

16 Aadland E, Schrumpf E, Fausa O, Elgjo K, Heilo A, Aakhus T, Gojne E. Primary sclerosing cholangitis: a long-term follow-up study. Scand $\mathcal{F}$ Gastroenterol 1987; 22: 655-64.

17 Olsson R, Danielsson Å, Järnerot G, Lindström E, Lööf L, Rolny $\mathrm{P}$, et al. Prevalence of primary sclerosing cholangitis in patients with ulcerative colitis. Gastroenterology 1991; 100: $1319-23$

18 Rasmussen HH, Fallingsborg J, Freund L, Tage-Jensen U, Kruse V, Rasmussen SN. Primary sclerosing cholangitis in patients with ulcerative colitis. Scand f Gastroenterol 1992; 27: 732-6.

19 Broomé U, Glaumann H, Hellers G, Nilsson B, Sörstad J, Hultcrantz R. Liver disease in ulcerative colitis: an epidemiological and follow up study in the county of Stockholm. Gut 1994; 35: 84-9.

20 Mattsson B, Wallgren A. Completeness of the Swedish Cancer Register. Nonnotified cancer cases recorded on death certificates in 1978. Acta Radiol Oncol 1984; 23: 305-13.

21 Kalbfleisch JP, Prentice RL. The statistical analysis of failure time data. New York: Wiley, 1980.

22 Gyde SN, Prior P, Thomson H, Waterhouse JAH, Allan RN, Stevens A, et al. Colorectal cancer in ulcerative colitis: short study of primary referrals from three centers. Gut 1988; 29: 206-17.

23 Devroede GJ, Taylor WF, Sauer WG, Jackman RI, Stickler GB. Cancer risk and life expectancy of children with ulcerative colitis. N Engl f Med 1971; 285: 17-21.

24 Ahnen DJ. Controlled clinical trials: the controls are the key. Gastroenterology 1996; 110: 628-30.

25 Gyde SN, Prior P, Thomson H, Waterhouse JAH, Allan RN. Survival of patients with colorectal cancer complicating ulcerative colitis. Gut 1984; 25: 228-31.

26 Cangemi JR, Wiesner RH, Beaver SJ, Ludwig J, MacCarty RL, Dozois RR, et al. Effect of proctocolectomy for chronic ulcerative colitis on the natural history of primary sclerosing cholangitis. Gastroenterology 1989; 96: 790-4.

27 Higashi H, Yanaga K, Marsh JW, Tzakis A, Kakizoe S, Starzl TE. Development of colon cancer after liver transplantation for primary sclerosing cholangitis associated with ulcerative colitis. Hepatology 1990; 11: 477-80. 